This document is the Accepted Manuscript version of a Published Work that appeared in final form in J. Chem. Phys. 133, 144510 (2010), copyright (c) American Institute of Physics after peer review and technical editing by the publisher. To access the final edited and published work see

$$
\text { jcp.aip.org }
$$




\title{
Analysis Of Localization Sites for An Excess Electron In Neutral Methanol Clusters Using Approximate Pseudopotential Quantum- Mechanical Calculations
}

\author{
Letif Mones ${ }^{\mathrm{a}}$, Peter J. Rossky ${ }^{\mathrm{b}}$ and László Turi ${ }^{\mathrm{a}, 1}$ \\ ${ }^{a}$ Eötvös Loránd University, Department of Physical Chemistry, Budapest 112, P. O. Box 32, \\ H-1518, Hungary \\ ${ }^{\mathrm{b}}$ Department of Chemistry and Biochemistry and Institute for Computational Engineering and \\ Sciences, \\ University of Texas at Austin, Austin, TX 78712
}

We have used a recently developed electron-methanol molecule pseudopotential in approximate quantum mechanical calculations to evaluate and statistically analyze the physical properties of an excess electron in the field of equilibrated neutral methanol clusters $\left(\left(\mathrm{CH}_{3} \mathrm{OH}\right)_{\mathrm{n}}, n=50-500\right)$. The methanol clusters were generated in classical molecular dynamics simulations at nominal $100 \mathrm{~K}$ and $200 \mathrm{~K}$ temperatures. Topological analysis of the neutral clusters indicates that methyl groups cover the surface of the clusters almost exclusively, while the associated hydroxyl groups point inside. Since the initial neutral clusters are lacking polarity on the surface and compact inside, the excess electron can barely attach to these structures. Nevertheless, most of the investigated cluster configurations do support weakly stabilized cluster anion states. We find that similarly to water clusters, the preexisting instantaneous dipole moment of the neutral clusters binds the electron. The localizing

\footnotetext{
${ }^{1}$ E-mail: turi@chem.elte.hu, fax: (36)-1-372-2592 molet@enzim.hu, fax: (36)-1-372-2592

rossky@mail.utexas.edu, fax: (1)-512-471-1624
} 
electrons occupy diffuse, weakly bound surface states that largely engulf the cluster although their centers are located outside the cluster molecular frame. The initial localization of the excess electron is reflected in its larger radius compared to water due to the lack of free $\mathrm{OH}$ hydrogens on the cluster surface. The stabilization of the excess electron increases, while the radius decreases monotonically as the clusters grow in size. Stable, interior bound states of the excess electron are not observed to form neither in finite size methanol clusters nor in the equilibrium bulk. 


\section{Introduction}

Finite size solvated electron systems have been intensively studied, both experimentally ${ }^{1,2,3,4,5,6,7}$ and theoretically, ${ }^{8,9,10,11,12}$ in the last few years. The main motivation of the scientific activity stems from the fact that cluster anions represent the transition from gaseous species to the bulk solvated electron. Although much of the interest has been concentrated on the aqueous case, the extension of the investigations to other polar solvents is a logical approach to reach a more complete understanding of the molecular level details underlying the experimental observations.

Compared to the water very few studies have examined methanol cluster anions experimentally. ${ }^{5,6,7}$ Very recently, the Neumark group performed photoelectron imaging measurements on negatively charged methanol clusters. ${ }^{6,7}$ The observation of two different sequences in the excess electron vertical detachment energy (VDE) with cluster size suggests the appearance of two different types of cluster isomers. According to a tentative explanation, the more strongly bound feature was assigned to structures that internally solvate the excess electrons (interior-bound excess electron states) in analogy to the bulk solvated electron. The significantly more weakly bound signals, on the other hand, may indicate the presence of an alternative binding motif, surface-bound excess electrons. Nevertheless, a more certain identification of these two methanol cluster anion classes is still to be resolved. We also note that water cluster anion experiments ${ }^{3,4}$ indicate more complex pattern with at least three classes showing systematic variations in VDE with size.

Theoretical studies on methanol cluster anions are scarce. The well-known reason is that all-electron (or, at least, all-valence-electron) quantum calculations are still expensive, and are limited to relatively small system size (i.e. few tens of atoms). The only quantum chemistry calculations on small methanol cluster anions that we are aware of were performed using density functional methods with relatively modest basis sets. ${ }^{13}$ Mixed quantum-classical 
molecular dynamics (QCMD) simulations that treat the excess electron quantum mechanically and the solvent bath classically, offer a viable alternative. ${ }^{14,15,16}$ This approach has provided a molecular level picture of the bulk solvated electron in methanol $17,18,19,20,21,22,23$ that is consistent with experimental observations. ${ }^{24,25,26,27,28,29,30}$ Despite the general qualitative agreement of experiment and theory, it was found that the measured position and the halfwidth of the optical spectrum of the solvated electron in methanol ${ }^{26}$ is not satisfactorily predicted in simulations. ${ }^{18}$ To remedy this inadequacy, a new electron-methanol molecule pseudopotential has been developed recently using static exchange pseudopotential theory. ${ }^{31}$ Application of the new potential in QCMD simulation of an excess electron in bulk methanol was notably improved resulting in good general agreement with the experimental observations. In particular, the computed structural properties are in excellent agreement with those inferred from the electron spin echo measurements of Kevan. ${ }^{25}$ Although the high energy tail of the spectrum is still underestimated, the position of the absorption maximum is properly reproduced in the new model.

In the present paper, we turn our attention to the simulation of methanol cluster anions. In particular, we take the logical first step and characterize the electron localization properties of neutral equilibrated methanol clusters. The following analysis is similar to that we performed on neutral equilibrated water clusters, ${ }^{11}$ and can be considered as the simplest physical scenario that models the preparation of cluster anions. Here, we essentially mimic the initial conditions of low energy electron attachment to pre-existing equilibrium methanol clusters by adding an electron to equilibrated neutral clusters. Although, our knowledge of the experimental conditions (i.e. whether the experimental clusters are at equilibrium) is clearly limited, the equilibrium hypothesis still provides a well-defined, reasonable framework to study the influence of the basic variables (cluster size and temperature) on the attachment 
process. Comparison with the aqueous case can also give additional information on the mechanistic details (and possible differences) of electron solvation in different solvents.

\section{Methods}

To investigate the binding energy of the electron to the neutral clusters, we performed classical molecular dynamics simulations of 11 different clusters in the size range from 50 to 500 equidistantly spaced on the $n^{1 / 3}$ scale ( $n$ is the number of methanol molecules in the given cluster). The time step of the simulations was $1.0 \mathrm{fs}$ and the velocity Verlet integration algorithm $^{32}$ was applied. The 6 site OPLS (Optimized Parameters for Liquid Simulations) ${ }^{33}$ force field was used and all non-bonded interactions were considered. The cluster properties and binding energies were investigated at two temperatures: 100 and $200 \mathrm{~K}$ (at $300 \mathrm{~K}$ the clusters tend to vaporize). The clusters were carefully equilibrated at these temperatures: each cluster was gradually heated from $1 \mathrm{~K}$ to the target temperature $(100$ or $200 \mathrm{~K})$ in a 50 ps simulation using the Berendsen thermostat ${ }^{34}$ with $\tau=0.4$ ps bath coupling parameter. At the target temperature an additional 200 ps long relaxation trajectory was generated using the microcanonical (NVE) ensemble with a Maxwell-Boltzmann velocity distribution regularly applied every 5 ps. After the relaxation, equilibrium NVE simulations were carried out at 100 and $200 \mathrm{~K}$ ( $2 \mathrm{~ns}$ and $1 \mathrm{~ns}$ long trajectories, respectively). The configurations were recorded every $100 \mathrm{fs}$ (20000 and 10000 configurations altogether, respectively).

To find possible interior electron localization sites, we also performed classical simulations of bulk methanol at temperatures of 100, 200 and $300 \mathrm{~K}$. The solvent bath consists of 200 methanol molecules in a cubic simulation cell using periodic boundary conditions. Since the densities of the OPLS methanol bulk were unknown at lower temperatures, we performed $1 \mathrm{~ns}$ isobaric-isothermal (NPT) simulations at all three temperatures to determine these quantities. At $300 \mathrm{~K}$ we found the average density $0.775 \mathrm{~g}$ 
$\mathrm{cm}^{-3}$ that is very close to the literature value $\left(0.779 \mathrm{~g} \mathrm{~cm}^{-3}\right) .{ }^{33}$ The simulated average densities were found to be 0.867 and $0.924 \mathrm{~g} \mathrm{~cm}^{-3}$ for the $200 \mathrm{~K}$ and $100 \mathrm{~K}$ simulations, respectively. The equilibration process for the methanol baths was the following. The systems were gradually heated from $1 \mathrm{~K}$ to the target temperatures in a $50 \mathrm{ps}$ simulations using the Berendsen thermostat and $\tau=0.4$ ps. At the target temperatures 400,400 and $1400 \mathrm{ps}$ canonical (NVT) simulations were carried out for the systems with $300 \mathrm{~K}, 200 \mathrm{~K}$ and $100 \mathrm{~K}$ temperatures, respectively. Finally, the equilibrium configurations were collected from an additional $1 \mathrm{~ns}$ canonical simulation (10000 snapshots, similarly to the clusters). In the bulk simulations, the calculation of the interactions was not corrected for the long-range interactions beyond the minimum image convention.

The interaction energies between the excess electron and the neutral systems were computed in subsequent single point quantum mechanical calculations using each of the collected configurations. The interaction between the quantum particle (electron) and the classical part (methanol cluster or bath) of the system was modeled using the recently developed electron-methanol pseudopotential. ${ }^{31}$ The present pseudopotential was derived with a similar protocol as a previous electron-water molecule pseudopotential. ${ }^{35,36}$ The electronwater pseudopotential has been successfully applied in a series of QCMD simulations on an excess electron bound to neutral water clusters, ${ }^{11}$ relaxed water anion clusters, ${ }^{10,12}$ electron solvation on water/air interfaces ${ }^{37}$ and in bulk water. ${ }^{36}$ It was also shown, ${ }^{11}$ however, that the binding energies of an excess electron to neutral water clusters are systematically underestimated. In fact, similarly to the aqueous case, ${ }^{38,39}$ the present pseudopotential is likely to mainly neglect electron-molecule dispersion interactions. Thus, one might expect that the potential surface at smaller electron-cluster interaction energies will be somewhat too weak, with the present pseudopotential. Nevertheless, our findings for the electron-water cluster 
system suggest that the pseudopotential based method can be used with confidence in a wide range of stabilization energies for qualitative purposes, such as in the present work.

The excess electron was described by a plane-wave basis similarly to that in Ref.31. We performed the quantum mechanical calculations in two consecutive steps. In the first step, we employed a box length of $50 \AA$ and $32 \times 32 \times 32$ grid points centered on the centre of mass of electron. In this calculation, the box size was large enough to capture the lowest energy electron localization site but, at the same time, it provided only a rough estimate of the binding energy due to the relatively large distance between the grid points. In a second step, we used a smaller box length of $25 \AA$ with $32 \times 32 \times 32$ grid points and constrained the center of the grid to the center of mass of the electron from the previous calculation. The convergence of the energy eigenvalues with respect to the smaller grid box was verified by calculating the binding energies for the configurations of the lowest energy (1\% of the collected configurations) using $64 \times 64 \times 64$ grid points. In the bulk simulations, $32 \times 32 \times 32$ grid points were used in the simulation box. The Schrodinger equation was solved by an iterative and block Lanczos procedure. ${ }^{40}$

\section{Results}

\section{A. Structural properties of the neutral methanol clusters}

Before the calculation of the binding energies, we have quantitatively analyzed two structure-related properties of the classical neutral clusters, the dipole moment and the relative proportion of the different atoms on the cluster surfaces. These properties, as we will show, are strongly related to the propensity of the clusters to bind the excess electron. We performed the analysis on 20000 and 10000 neutral configurations collected from the classical molecular dynamics runs at 100 and $200 \mathrm{~K}$, respectively. 
First, we notice that the dipole moment of the single all atom model methanol molecule, $2.23 \mathrm{D}$, is significantly higher than the gas phase value of $1.7 \mathrm{D}$. This value is almost identical to the three-site OPLS model $(2.22 \mathrm{D}) .{ }^{41}$ As was shown in the case of electron binding to neutral water clusters, ${ }^{11}$ the electron stabilization strongly depends on the instantaneous dipole moment of the parent cluster. The net dipole moments of the clusters are shown in Figure 1 at $100 \mathrm{~K}$ and $200 \mathrm{~K}$. As is expected, at both temperatures the dipole moment increases with the size of the clusters. The major difference is that the mean dipole moments have greater magnitude when fluctuations are larger, at $200 \mathrm{~K}$ than at $100 \mathrm{~K}$. Molecular dynamics simulations showed for medium size water clusters $(n \leq 100)$ that at a temperature slightly higher than examined here $(233 \mathrm{~K})$, the total dipole moment follows a $\mu \propto \sqrt{n}$ size dependence arising from the random fluctuations of the constituting molecular dipoles. ${ }^{42}$ Here we observe similar size dependence for the methanol clusters at $200 \mathrm{~K}$. Due to the smaller available kinetic energy of the molecules at $100 \mathrm{~K}$, the reorientation of the dipoles becomes hindered resulting in lower net dipole moment magnitudes. It is also clear that the curve at $100 \mathrm{~K}$ is less continuous and has larger deviations. Here we cannot rule out the possibility that at lower temperatures the timeframe of the simulations may not be sufficient to completely sample the phase space. It is also important to observe that the total dipole moments of the methanol clusters are similar to those of water clusters of the same size. ${ }^{11}$ It was shown that the initial binding strength of an excess electron to equilibrated water clusters is mainly determined by the dipole moment of the cluster. ${ }^{11}$ Based on this finding, one may expect similar initial electron binding strength by the methanol clusters.

An important difference may, however, appear between the behavior of water and methanol clusters. That is because methanol incorporates a non-polar group. The position and orientation of the non-polar methyl group need also to be considered in the analysis. To do this, we estimated the relative proportion of the $\mathrm{O}, \mathrm{H}(\mathrm{O}), \mathrm{C}$ and $\mathrm{H}(\mathrm{C})$ atoms on the cluster 
surfaces using the concept of atomic accessibilities. The atomic accessibilities were evaluated using the "Naccess" program ${ }^{43}$ which is based on the method of Lee and Richards. ${ }^{44}$ In the calculations we used $1.72 \AA, 1.91 \AA$ and $1.49 \AA$ for the atomic radii for oxygen, carbon and hydrogen atoms (both $\mathrm{H}(\mathrm{C})$ and $\mathrm{H}(\mathrm{O})$ atoms), respectively. For the probe radius, we kept the default value (1.4 $\AA$ ). We considered an atom to be at the surface when its accessible surface area was greater then $8.0 \AA^{2}$. This value proved to be large enough to filter out the atoms inside the clusters. For each configuration we simply counted the atoms on the surfaces and performed the statistics over the configurations. The proportion of the $\mathrm{O}, \mathrm{H}(\mathrm{O})$ and $\mathrm{H}(\mathrm{C})$ atoms at the two temperatures are shown in Figure 2. Since the carbon atom is enveloped by three $\mathrm{H}(\mathrm{C})$ atoms, we did not include carbon in the analysis. The figure illustrates that the cluster surfaces are covered mainly by methyl hydrogen atoms (more than 90\%) and, in smaller proportion, by oxygen atoms $(5-8 \%)$. We notice that at $200 \mathrm{~K}$ there are somewhat less methyl hydrogen atoms on the surface but the decrease in the proportion is due to the few $\%$ increase of the surface oxygen atoms. The hydroxyl hydrogen contribution remains less than $1 \%$ in all clusters independently of size and temperature. This indicates that there are no "free" surface $\mathrm{OH}$ groups to provide potential localization sites for the excess electron. We also calculated the radial distributions of the different atoms measured from the center of mass of the cluster. We find that the descent of curves of the oxygen and hydroxyl hydrogen atoms always precedes the carbon and methyl hydrogen ones by at least $1 \AA$ (Figure 3 ) which also supports the previous findings.

The fact that the clusters' surface is mostly covered by methyl hydrogen atoms implies that dipole moment of methanol molecules on the surface points inward to the clusters. To investigate the difference of the dipole distribution between the inner space and surface of the cluster, we calculated the radial distribution of the cosine of the angle between the dipole moment vector and the position vector pointing from the center of mass of the molecule to the 
center of mass of the cluster (Figure 4). The average value of the $\cos (\theta)$ as a function of the radial distance measured from the center of mass of the cluster is roughly 0 (after a transient region within $5 \AA$, which is poorly sampled). This means that there is no preference of the molecules' dipoles inside the cluster and therefore the statistical average is 0 . As the distance reaches the surface of the cluster, the $\cos (\theta)$ function significantly decreases to $-0.15--0.2$ at $200 \mathrm{~K}$ (Figure 4) and -0.3 at $100 \mathrm{~K}$ corresponding to $\sim 100^{\circ}$ and $\sim 108^{\circ}$, respectively. Similar angle distribution for the $\mathrm{O}-\mathrm{C}$ bond predicts $\sim 35^{\circ}$ at $100 \mathrm{~K}$, and $\sim 50^{\circ}$ at $200 \mathrm{~K}$. These data indicate that the methyl-groups predominantly project out from the cluster to the vapor. It is clear that the surface layer of the methanol clusters is mostly hydrophobic. This qualitative statement agrees with previous observations on classical methanol clusters. ${ }^{45,46}$ Before turning to the analysis of the electron binding to methanol clusters, we note that we have also analyzed possible correlations between the shape of the clusters and the net dipole moment. The shape of the clusters was characterized by the asymmetry parameter (or first flattening)

$$
f=\frac{I_{a}-I_{b}}{I_{a}}
$$

where $I_{a}$ is largest and $I_{b}$ is the smallest principal moment of inertia of the cluster. We find that the asymmetry parameter monotonically decreases (from $\sim 0.3$ to $\sim 0.1$ ) as the clusters grow, but no correlation is evident between the asymmetry and the total dipole moment.

\section{B. Binding properties of the excess electron to neutral methanol clusters}

We report the converged quantum mechanical calculations that employ a grid box length of $25 \AA$ with $32 \times 32 \times 32$ grid points. For the present purposes, we consider the electron to be "bound" to the cluster if its ground state energy is lower than that of a free electron but computed using the same grid as those in the presence of the cluster, $0.00463 \mathrm{eV}$. We observe that at both temperatures, the electron is bound in at least $87 \%$ of the configurations. Figure 5 
plots the average binding energies of the excess electron to the neutral methanol clusters at $100 \mathrm{~K}$ and $200 \mathrm{~K}$. Clearly, the initial ground state energy of the electron is lower at the higher temperature, consistent with the temperature dependence of the dipole moment and similar to observations in the case of water. ${ }^{11}$ The ground state energies in methanol at $200 \mathrm{~K}$ are also quantitatively similar to those found in the water case, consistent with the similarity in the total dipole moments. For the methanol, a linear relationship between the dipole moment and the ground state energy also holds well in accord with simple electrostatics (Figure 6). Figure 5 also shows the result of the analysis for $1 \%$ of the configurations with the most stable ground state energies. The tendency is similar to the full set of bound states, except that the energies are now more negative by more than a factor of two, extending down to about -0.35 $\mathrm{eV}(n=500, T=200 \mathrm{~K})$. These most stable configurations demonstrate again the strong correlation between ground state energy and instantaneous dipole moment: the average dipole moment for the selected, more strongly bound configurations is approximately double of the average dipole moment of all the analyzed configurations (See Fig. 1). It is also notable that the binding energies of Figure 5 are qualitatively similar to those measured by the Neumark group for the more weakly bound cluster type. ${ }^{6}$

Now we turn to the electron localization, which we characterize by geometric parameters, namely the radius of the cluster $\left(r_{c}\right)$, the radius of gyration of the electron $\left(r_{e}\right)$ and the distance between the centers of mass of the cluster and the electron $(R)$. We use the same definitions for the interior and surface states as were employed for water clusters. ${ }^{10,11,12}$ We consider the electron to localize in the interior of the cluster if $R+r_{e}<r_{c}$, while for surface states $R \geq r_{c}$. For all examined configurations, we did not find a single case with interior excess electron state. The observed surface states, however, are all very diffuse, with radius of gyration of 9-11 A for our more refined grid, depending on the cluster size. Since these values 
are greater than the radius of gyration of a uniform sphere with radius of $l_{b o x} / 2\left(r_{g}=\sqrt{\frac{3}{5}} \frac{l_{b o x}}{2}\right)$, these diffuse states do not reflect the real spread of the electron. For this reason, we returned to our coarser grid calculations using the grid box with length of $50 \AA$. Figure 7 illustrates the diffuse character of the initial excess electron states for three different size clusters at $200 \mathrm{~K}$. The radius of gyration visibly shrinks with increasing cluster size. The calculated radii for all clusters are collected in Figure 8. Clearly, the cluster size, the dipole moment of the cluster and the average ground state energy all correlate strongly with the electronic radius of gyration. We also notice that while the ground state energies of the electron are very close in the case of water and methanol clusters of the same size, the radius of gyration is somewhat larger in methanol clusters at $200 \mathrm{~K}$. For example, for the $n=500$ cluster, the average radius is around $10 \AA$ in water, ${ }^{11}$ while $12 \AA$ in methanol. It is likely that the difference originates from the topological properties of the cluster surfaces. Although in both cases the clusters have large enough dipole moment to bind the electron, the surface of the methanol clusters is occupied mostly by methyl hydrogens that accommodate the electron less favorably than the hydroxyl hydrogens on the water cluster surfaces. Nevertheless, we can conclude, that the surface topology does not dominate the initial electron binding properties; it is the dipole moment that determines the strength of the binding.

In the next step, we examined the alternative electron localization route, in the interior of the solvent. To facilitate the comparison with surface state localization, we excluded the possibility of surface localization by examining the electron attachment in bulk methanol. Due to the periodic boundary conditions, the electron has no alternative but localize in the bulk. The average ground state energies for the 10000 sampled configurations $(1.67,1.37$ and 0.92 $\mathrm{eV}$ at $100 \mathrm{~K}, 200 \mathrm{~K}$ and $300 \mathrm{~K}$, respectively) indicate that the electron does not find favorable cavities in liquid methanol. The electron radii (8.0, 7.2 and $6.1 \AA$ at $100 \mathrm{~K}, 200 \mathrm{~K}$ and $300 \mathrm{~K}$, 
respectively) are significantly smaller than in even the most strongly bound surface state localization, but the smaller spatial extent is the consequence of the strongly repulsive potential energy landscape of the bulk methanol, the electron ground state energies are much higher in the bulk solvent than on the cluster surfaces. This finding is generally similar to that observed for liquid water, ${ }^{11}$ although it is known that cavities which bind the electron exist in water. ${ }^{47}$ Comparison with the aqueous case also shows that the stabilization in equilibrium bulk methanol is more unfavorable compared with bulk water. ${ }^{11}$ Nevertheless, as the temperature increases the ground state energy becomes lower. Here two reasons likely can play a role. First, at higher temperature the density is lower which presumably promotes the formation of larger cavities, where the electron can find less unfavorable localization. Second, at higher temperature the relative proportion of "free" (not involved in H-bond) $\mathrm{OH}$ hydrogens is larger which would also facilitate electron stabilization.

\section{Discussion and Conclusions}

In the work presented, we have analyzed the electron binding properties of neutral equilibrated methanol clusters using a combination of classical molecular dynamics simulations and approximate pseudopotential based quantum mechanical calculations. The analysis showed very similar behavior for methanol clusters and water clusters, basic electrostatics dominating the phenomenon. The initial electron binding strength and the degree of localization are largely determined by the dipole moment of the neutral clusters. Since the dipole moments are similar in water and methanol clusters at the same cluster size, the electron binding energies are similar in both systems. The electron localization takes place on the cluster surfaces in weakly bound, diffuse surface electronic states. We find that the shape of the clusters do not correlate with binding strength of the electron significantly. Thus, the methanol clusters having about the same dipole moments as for water clusters but 
possessing inert, methyl hydrogen atom covered surfaces, bind the electron similarly to water clusters. The difference in the surface properties (inert methanol cluster surface vs. polar water surface with $\sim 20 \%$ of surface water molecules having one free $\mathrm{OH}$ that point to the vapor $^{48}$ ) is reflected only in a slight difference of the radius of the initially bound excess electron on methanol vs. water clusters. We also investigated electron localization in the interior of bulk methanol and compared it to the stabilization on methanol cluster surfaces. We found that the excess electron unquestionably prefers localization on the cluster surfaces. While the initial stabilization on the surfaces is energetically weakly favorable, this is not the case for interior localization. An important question arises, as to what happens with the clusters in their subsequent nuclear relaxation. If the kinetic energy of the molecules is so low, that it prevents significant rearrangement of the surface, the system can remain kinetically trapped in weakly stabilized dipole bound surface states. We speculate that this scenario may correspond to the weakly bound feature of Neumark's measurements. ${ }^{6}$ If, on the other hand, the clusters gain enough energy to break the interior hydrogen-bonding system, and open the apolar surface, then either more strongly bound surface solvated electron states can form, or the excess electron can penetrate into the bulk to eventually form a strongly bound bulk solvated electron. We are presently examining the relaxation trajectories of methanol cluster anions, in order to answer these questions in the near future.

\section{Acknowledgements}

The work was supported by a grant to L. T. from the National Research Fund of Hungary (OTKA) under Contracts No. T049715 and IN82679. P. J. R. is grateful to the National Science Foundation (CHE-0910499) and the R. A. Welch Foundation (F-0019) for support of this work. 


\section{Figure Captions}

Figure 1. Average total dipole moment of neutral methanol clusters at $100 \mathrm{~K}$ (black) and 200 $\mathrm{K}$ (red). Averages of the total configurations are shown by squares while the averages for $1 \%$ of the configurations with the most stable electron binding energies are indicated by circles.

Figure 2. Proportion of the oxygen (square), the hydroxyl hydrogen (circle) and methyl hydrogen (triangle) atoms on the surface of the neutral methanol clusters at $100 \mathrm{~K}$ (black) and $200 \mathrm{~K}$ (red).

Figure 3. Average radial density function of the different atoms (oxygen - black, hydroxylhydrogen - red, carbon - green, methyl-hydrogen - blue) for four different clusters $(50,128$, 269 and 500 top to bottom) at $200 \mathrm{~K}$. The function is relative to the bulk one $\left(\rho_{0}\right)$ and the centre is the centre of mass of the cluster.

Figure 4. Radial average of the cosine of the angle between the dipole vector and the position vector pointing from the centre of mass of the molecule to the center of mass of the cluster at $200 \mathrm{~K}$ for four different size clusters: 50 (black), 128 (red), 269 (green) and 500 (blue).

Figure 5. Average electron binding energies at $100 \mathrm{~K}$ (black) and $200 \mathrm{~K}$ (red). The averages of the configurations with less energy than a free electron in the computational boundary are shown by squares while the average of the most stable $1 \%$ of the configurations is indicated by circles.

Figure 6. Linear relationship between the total dipole moment of neutral methanol clusters and the ground state energy of an excess electron attached to these clusters at $200 \mathrm{~K}$.

Figure 7. The most stable initial excess electron distributions on three different sized $(n=$ 128, 269 and 500, from the top) equilibrated methanol clusters at $200 \mathrm{~K}$. The electronic isosurface shown covers $80 \%$ of the excess electron density. 
Figure 8. Average radius of gyration of the electron attached to equilibrated neutral methanol clusters as a funtion of the size at $200 \mathrm{~K}$. 
Figure 1. Mones, Rossky and Turi

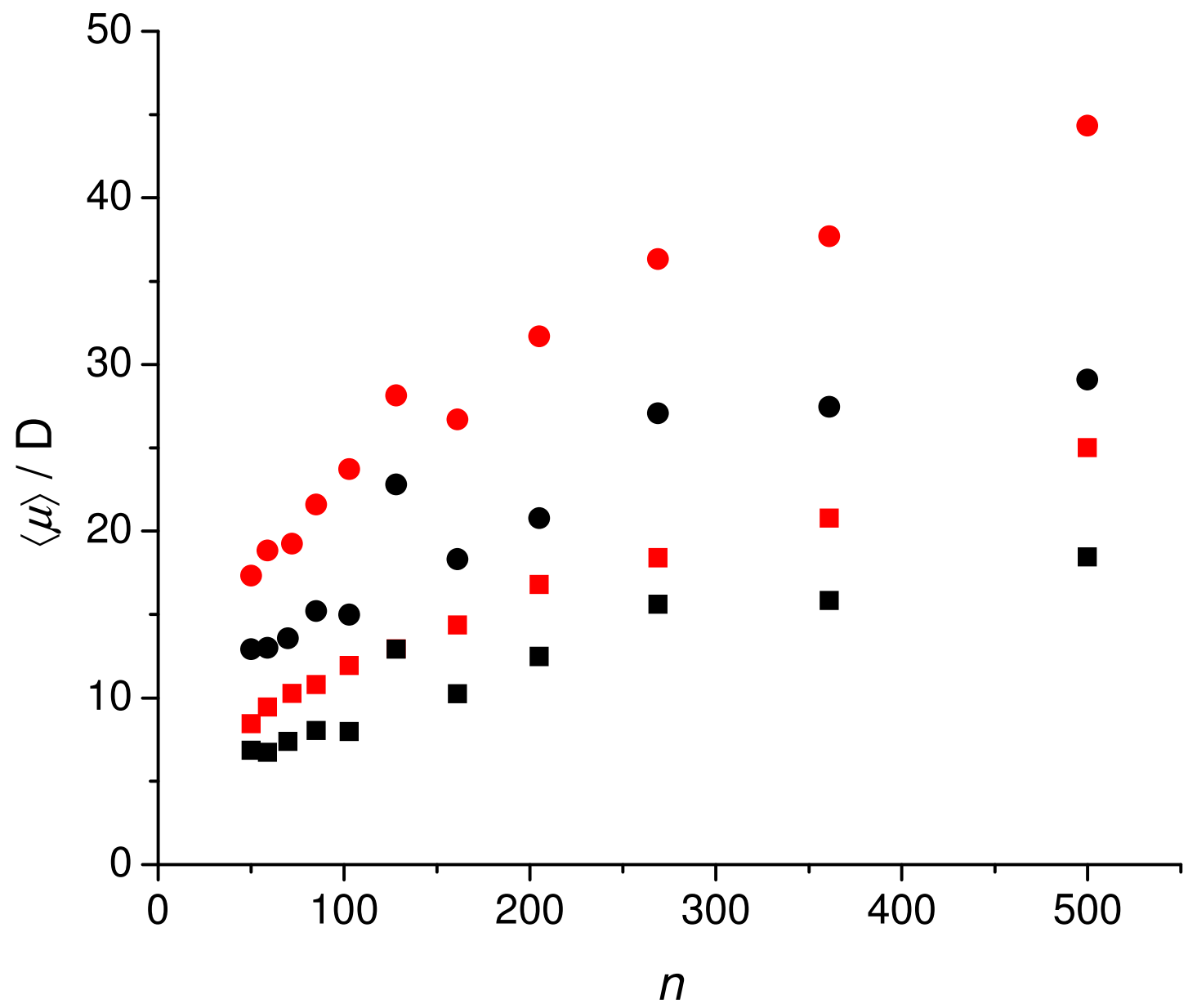


Figure 2. Mones, Rossky and Turi

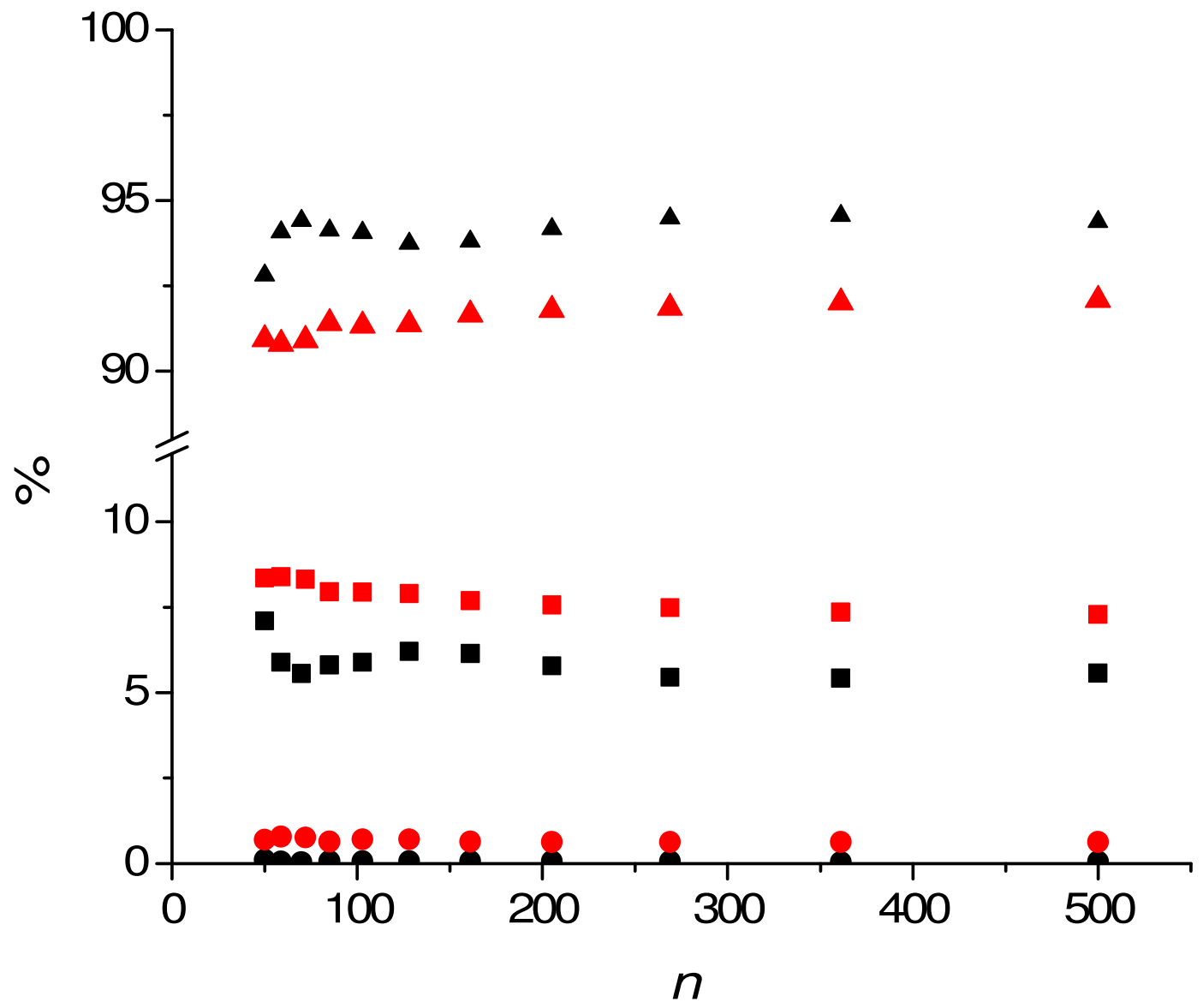


Figure 3. Mones, Rossky and Turi

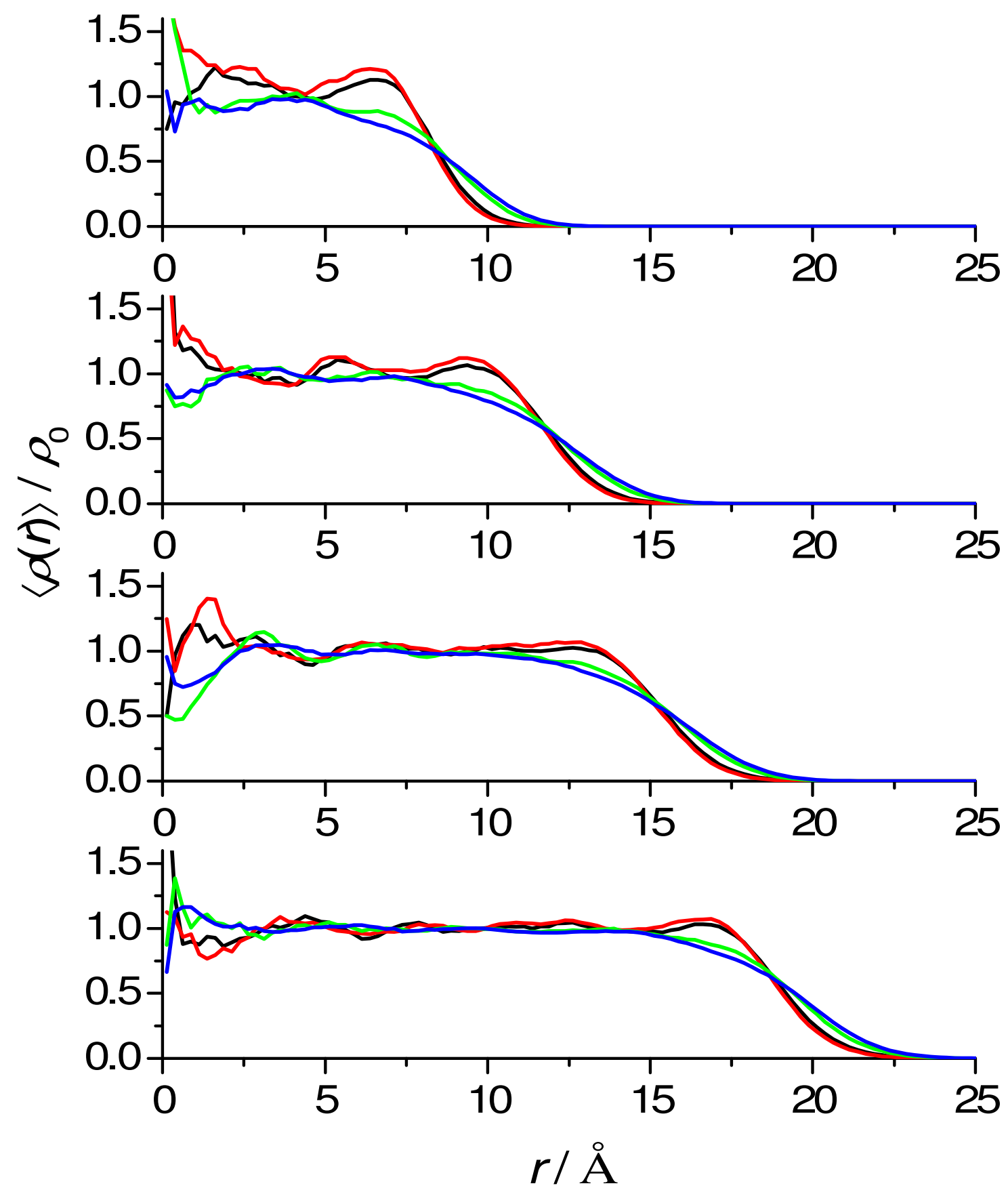


Figure 4. Mones, Rossky and Turi

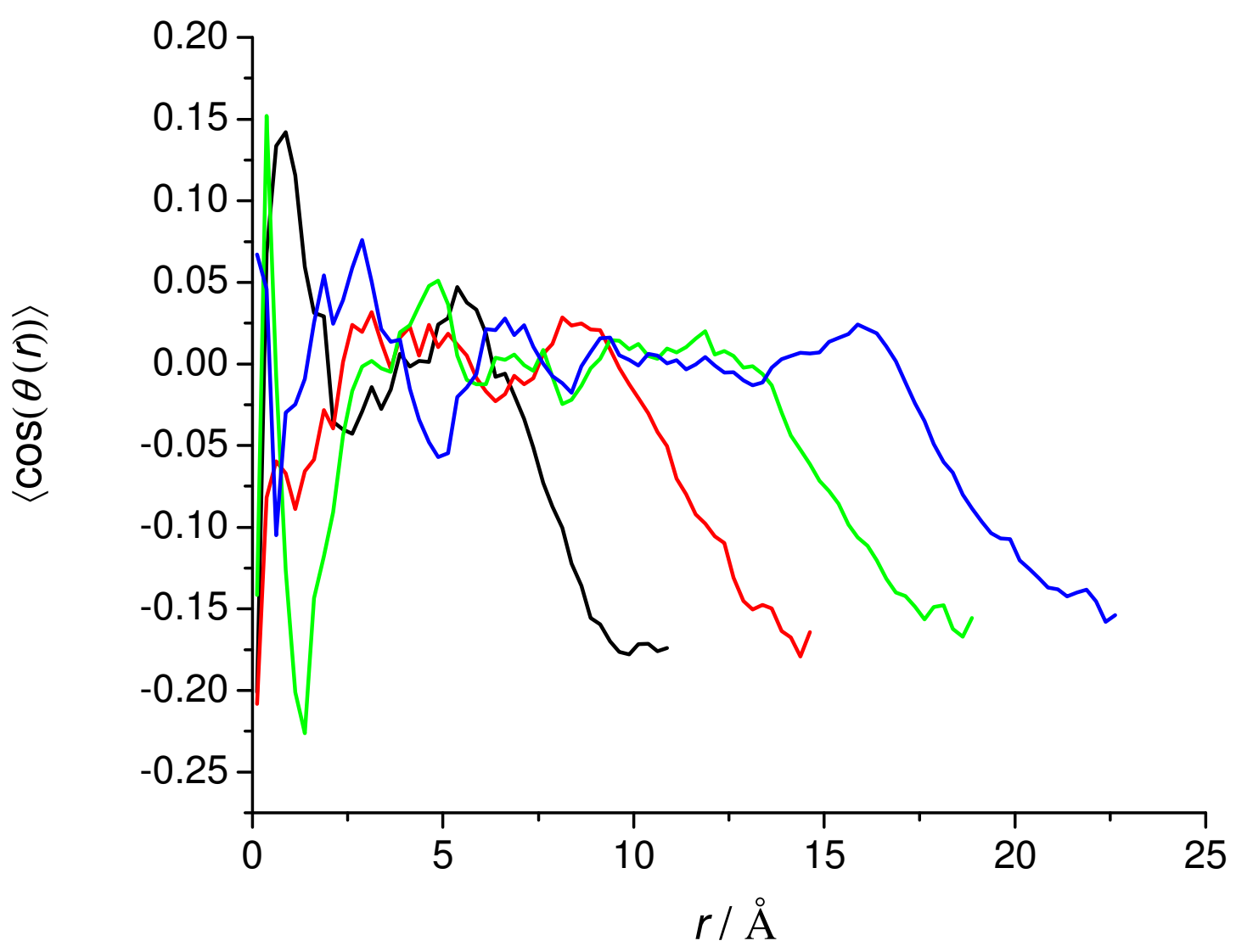


Figure 5. Mones, Rossky and Turi

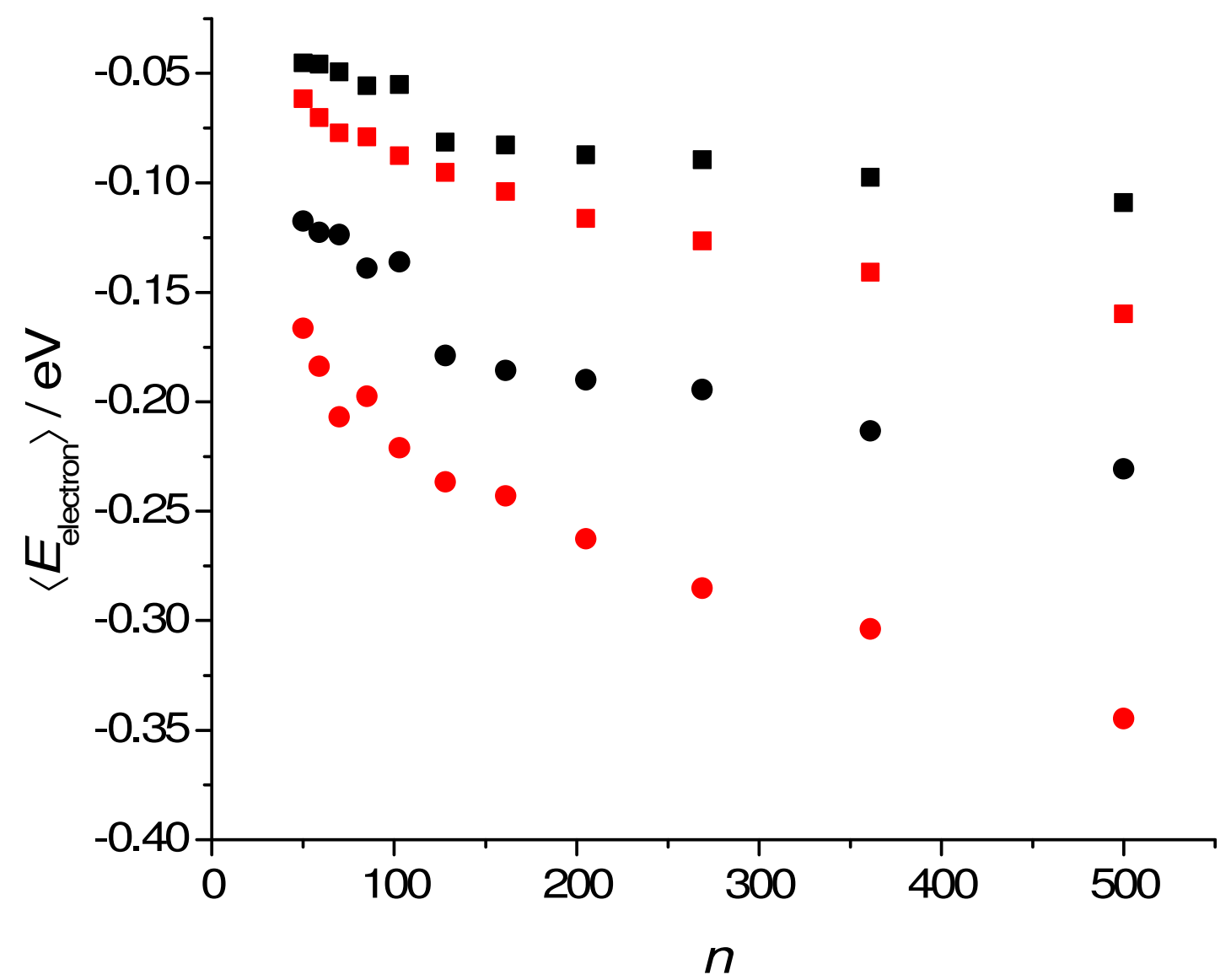


Figure 6. Mones, Rossky and Turi

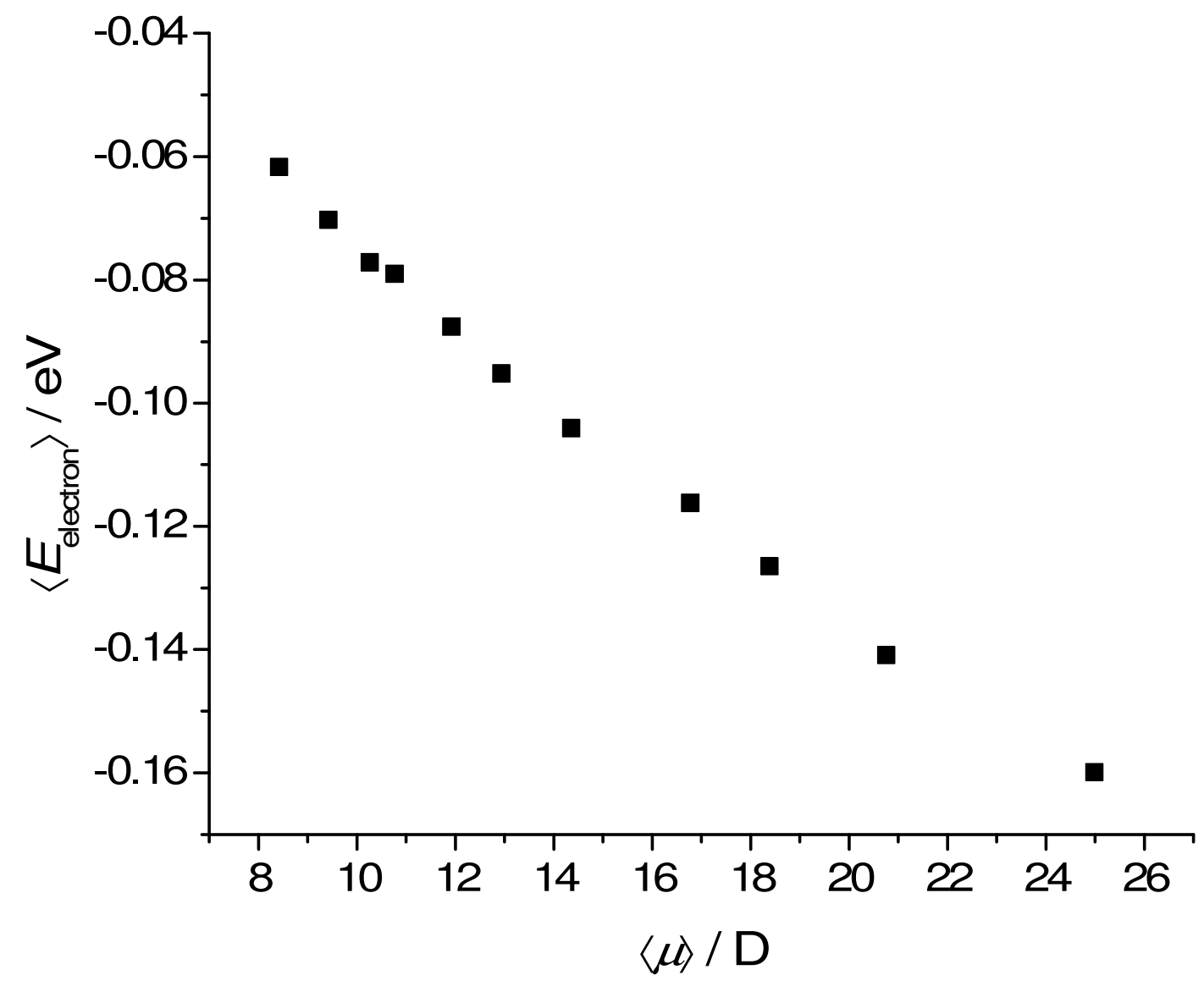


Figure 7. Mones, Rossky and Turi
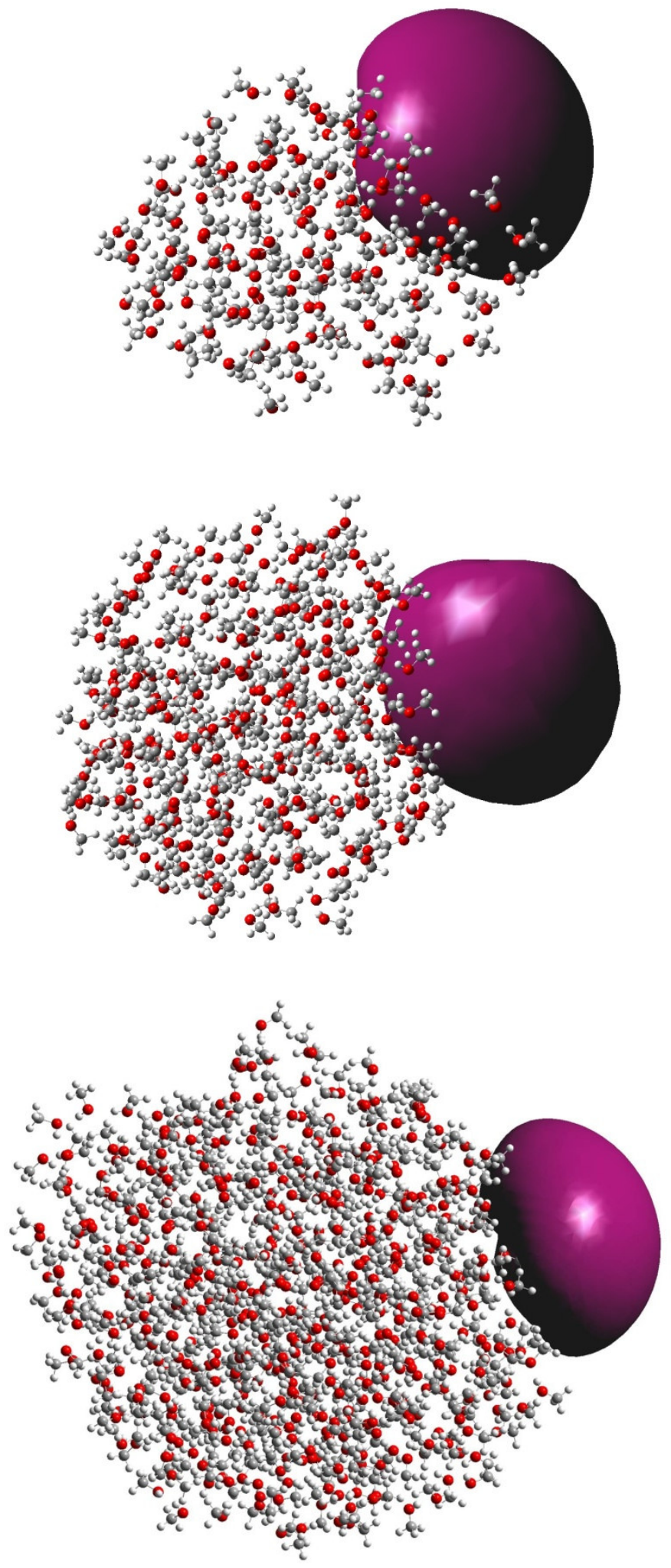
Figure 8. Mones, Rossky and Turi

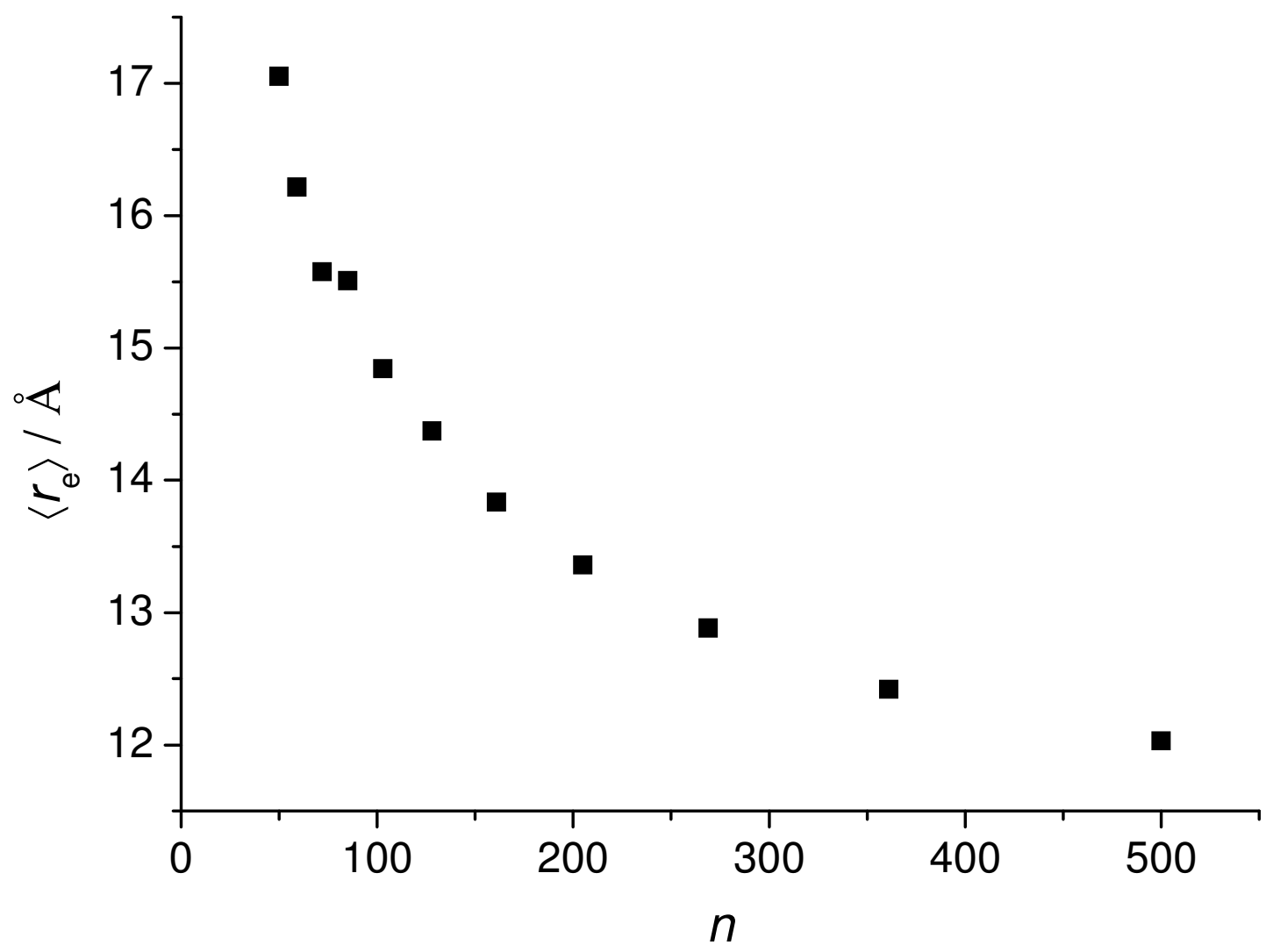




\section{References}

${ }^{1}$ D. H. Paik, I-R. Lee, D.-S. Yang, J. S. Baskin, and A. H. Zewail, Science 306, 672 (2004).

${ }^{2}$ N. I. Hammer, J.-W. Shin, J. M. Headrick, E. G. Diken, J. R. Roscioli, G. H. Weddle, and M. A. Johnson, Science 306, 675 (2004).

${ }^{3}$ J. R. R. Verlet, A. E. Bragg, A. Kammrath, O. Cheshnovsky, and D. M. Neumark, Science 307, 93 (2005).

${ }^{4}$ L. Ma, K. Majer, F. Chirot and B. v. Issendorff, J. Chem. Phys. 131, 144303 (2009).

${ }^{5}$ C. Desfrancois, H. Abdoul-Carime, N. Khefila, J. P. Schermann, V. Brenner and P. Millie, J. Chem. Phys. 102, 4952 (1995).

${ }^{6}$ A. Kammrath, J. R. R. Verlet, G. B. Griffin, and D. M. Neumark, J. Chem. Phys. 125, 171102 (2006).

${ }^{7}$ A. Kammrath, G. B. Griffin, J. R. R. Verlet, R. M. Young, and D. M. Neumark, J. Chem. Phys. 126, 244306 (2007).

${ }^{8}$ K. Yagi, Y. Okano, T. Sato, Y. Kawashima, T. Tsuneda and K. Hirao, J. Phys. Chem. A 112, 40 (2008).

${ }^{9}$ T. Frigato, J. VandeVondele, B. Schmidt, C. Schütte, and P. Jungwirth, J. Phys. Chem. A, 112, 6125 (2008).

${ }^{10}$ L. Turi, W.-S. Sheu, and P. J. Rossky, Science 309, 914 (2005).

${ }^{11}$ L. Turi, Á. Madarász, and P.J. Rossky, J. Chem. Phys. 125, 014308 (2006).

12 Á. Madarász, P. J. Rossky, and L. Turi, J. Chem. Phys. 130, 124319 (2009).

${ }^{13}$ L. Turi J. Chem. Phys. 110, 10364 (1999).

${ }^{14}$ Z. Kotler, E. Neria and A. Nitzan, Comput. Phys. Commun. 63, 243 (1991).

${ }^{15}$ F. A. Webster, P. J. Rossky and R. A. Friesner, Comput. Phys. Commun. 63, 494 (1991).

${ }^{16}$ A. Staib and D. Borgis, J. Chem. Phys. 103, 2642 (1995).

${ }^{17}$ J. Zhu and R. I. Cukier, J. Chem. Phys. 98, 5679 (1993). 
${ }^{18}$ L. Turi, A. Mosyak, and P. J. Rossky, J. Chem. Phys. 107, 1970 (1997).

${ }^{19}$ A. Mosyak, P. J. Rossky, and L. Turi, Chem. Phys. Lett. 282, 239 (1998).

${ }^{20}$ A. Mosyak, O. V. Prezhdo, and P. J. Rossky, J. Mol. Struct. 485-486, 545 (1999).

${ }^{21}$ P. Mináry, L. Turi, and P. J. Rossky, J. Chem. Phys. 110, 10953 (1999).

${ }^{22}$ L. Turi, P. Mináry, and P. J. Rossky, Chem. Phys. Lett. 316, 456 (2000).

${ }^{23}$ L. Turi and P. J. Rossky, J. Chem. Phys. 120, 3688 (2004).

${ }^{24}$ J. H. Baxendale and P. Wardman, J. Chem. Soc., Faraday Trans. 1 69, 584 (1973).

25 a) D. F. Feng and L. Kevan, Chem. Rev. 80, 1 (1980); b) L. Kevan, Radiat. Phys. Chem.

17, 413 (1981); c) L. Kevan, Chem. Phys. Lett. 66, 578 (1979).

${ }^{26}$ F. Y. Jou and G. R. Freeman, Can. J. Chem. 57, 591 (1979); F. Y. Jou and G. R. Freeman, J. Phys. Chem. 81, 909 (1977).

${ }^{27}$ X. Shi, F. H. Long, H. Lu and K. B. Eisenthal, J. Phys. Chem. 99, 6917 (1995).

${ }^{28}$ P. K. Walhout, J. C. Alfano, Y. Kimura, C. Silva, P. Reid and P. F. Barbara, Chem. Phys. Lett. 232, 135 (1995).

${ }^{29}$ C. M. Stuart, M. J. Tauber and R. A Mathies, J. Phys. Chem. A 111, 8390 (2007).

${ }^{30}$ H. Shen, N. Kurahashi, T. Horio, K. Sekiguchi and T. Suzuki, Chem. Lett. 39, 668 (2010).

${ }^{31}$ L. Mones and L. Turi, J. Chem. Phys. 132, 154507 (2010).

${ }^{32}$ W. C. Swope, H. C. Andersen, P. H. Berens, and K. R. Wilson, J. Chem. Phys. 76, 637 (1982).

${ }^{33}$ W. L. Jorgensen, D. S. Maxwell, and J. Tirado-Rives, J. Am. Chem. Soc. 118, 11225 (1996).

${ }^{34}$ H. J. C. Berendsen, J. P. M. Postna, W. F. van Gunsteren, A. di Nola, and J. R. Haak, J. Chem. Phys. 81, 3684 (1984).

${ }^{35}$ L. Turi, M.-P. Gaigeot, N. Levy and D. Borgis, J. Chem. Phys. 114, 7054 (2001).

${ }^{36}$ L. Turi, and D. Borgis, J. Chem. Phys. 117, 6186 (2002).

${ }^{37}$ Á. Madarász, P. J. Rossky, and L. Turi, J. Chem. Phys. 126, 234707 (2007). 
${ }^{38}$ F. Wang, and K. D. Jordan, Ann. Rev. Phys. Chem. 54, 367 (2003).

${ }^{39}$ K. D. Jordan, Science 306, 618 (2004).

${ }^{40}$ F. A. Webster, P. J. Rossky, and R. A. Friesner, Comput. Phys. Commun. 63, 494 (1991).

${ }^{41}$ W. L. Jorgensen, J. Phys. Chem. 90, 1276 (1986).

${ }^{42}$ A. E. Galashev and O. R. Rakhmanova, J. Struct. Chem. 46, 626 (2005).

${ }^{43}$ Naccess V2.1.1 - Atomic Solvent Accessible Area Calculations, S. Hubbard and J. Thornton 1992-6.

${ }^{44}$ B. Lee and F.M. Richards, J. Mol. Biol. 55, 379 (1971).

${ }^{45}$ D. Wright and M. S. El-Shall, J. Chem. Phys. 105, 11199, (1996).

${ }^{46}$ V. V. Zakharov, E. N. Brodskaya and A. Laaksonen, J. Chem. Phys. 109, 9487 (1998).

${ }^{47}$ K. A. Motakabbir, J. Schnitker and P. J. Rossky, J. Chem. Phys. 97, 2055 (1992).

${ }^{48}$ Q. Du, R. Superfine, E. Freysz and Y. R. Shen, Phys. Rev. Lett. 70, 2313 (1993). 